Article

\title{
Impact of Humidity on Quartz-Enhanced Photoacoustic Spectroscopy Based CO Detection Using a Near-IR Telecommunication Diode Laser
}

\author{
Xukun Yin 1,2,3, Lei Dong 1,2,*, Huadan Zheng 1,2, Xiaoli Liu 1,2, Hongpeng Wu 1,2, \\ Yanfang Yang 1,2, Weiguang Ma 1,2, Lei Zhang 1,2, Wangbao Yin 1,2, Liantuan Xiao 1,2 \\ and Suotang Jia 1,2 \\ 1 State Key Laboratory of Quantum Optics and Quantum Optics Devices, Institute of Laser Spectroscopy, \\ Shanxi University, Taiyuan 030006, China; yinxkvip@163.com (X.Y.); zhenghuadan@126.com (H.Z.); \\ liuxiaoli@163.com (X.L.); beijing2008whp@163.com (H.W.); 15735176489@163.com (Y.Y.); \\ mwg@sxu.edu.cn (W.M.); k1226@sxu.edu.cn (L.Z.); ywb65@sxu.edu.cn (W.Y.); xlt@sxu.edu.cn (L.X.); \\ tjia@sxu.edu.cn (S.J.) \\ 2 Collaborative Innovation Center of Extreme Optics, Shanxi University, Taiyuan 030006, China \\ 3 State Key Laboratory on Integrated Optoelectronics, Jilin University, Changchun 130012, China \\ * Correspondence: donglei@sxu.edu.cn; Tel.: +86-351-701-1045; Fax: +86-351-701-8927
}

Academic Editor: Vittorio M. N. Passaro

Received: 31 December 2015; Accepted: 22 January 2016; Published: 27 January 2016

\begin{abstract}
A near-IR CO trace gas sensor based on quartz-enhanced photoacoustic spectroscopy (QEPAS) is evaluated using humidified nitrogen samples. Relaxation processes in the CO-N $-\mathrm{N}_{2} \mathrm{O}$ system are investigated. A simple kinetic model is used to predict the sensor performance at different gas pressures. The results show that $\mathrm{CO}$ has a $\sim 3$ and $\sim 5$ times slower relaxation time constant than $\mathrm{CH}_{4}$ and $\mathrm{HCN}$, respectively, under dry conditions. However, with the presence of water, its relaxation time constant can be improved by three orders of magnitude. The experimentally determined normalized detection sensitivity for $\mathrm{CO}$ in humid gas is $1.556 \times 10^{-8} \mathrm{~W} \cdot \mathrm{cm}^{-1} / \mathrm{Hz}^{1 / 2}$.
\end{abstract}

Keywords: quartz enhanced photoacoustic spectroscopy; carbon monoxide; vibrational-to-translational (V-T) relaxation; near-IR telecommunication diode laser

\section{Introduction}

Photoacoustic spectroscopy (PAS) has been one of the most widely used spectroscopic techniques for trace gas detection in the past decades because of its advantages of high sensitivity, high selectivity and compact detection module. The principle of PAS is to detect the sound waves which are generated in the media upon absorption of modulated optical radiation. The optical radiation initially provides the vibrational excitation of molecules and then the excited states lose their energy by vibration-translation relaxation (V-T relaxation), resulting in heating and thermal expansion of the local gas. Periodic pressure waves, i.e., acoustic waves, are produced and subsequently detected by highly sensitive microphones. Quartz-enhanced photoacoustic spectroscopy (QEPAS), an alternative approach to PAS utilizing a quartz tuning fork (QTF) as a sharply resonant acoustic transducer instead of a microphone [1-3], has been widely applied to environmental monitoring, industrial process control, combustion processes analysis, and medical diagnosis [4-14]. Compared with the microphone-based PAS which employs the modulation frequency of $1-4 \mathrm{kHz}$ determined by the acoustic resonances modes of the photoacoustic cells, the QEPAS employs the higher modulation frequency of $\sim 32 \mathrm{kHz}$, corresponding to the oscillation frequency of the commercial QTFs. Such a high modulation frequency makes the QEPAS technique immune to $1 / f$ and environmental acoustic noise. However, a stringent condition for PAS which has to be taken into account is that the molecular relaxation time $\tau$ should be 
shorter than the modulation period, i.e., $\tau \ll 1 / f[15]$, because otherwise it can cause signal amplitude reduction or phase shifts of the photoacoustic signal when using QEPAS to detect the molecules with a slow V-T relaxation $[15,16]$. The $\mathrm{V}-\mathrm{T}$ relaxation processes of $\mathrm{CH}_{4}, \mathrm{HCN}$ and $\mathrm{CO}_{2}$ have been widely investigated using the QEPAS. However, the $\mathrm{V}$-T relaxation process of carbon monoxide $(\mathrm{CO})$ and the influence of $\mathrm{H}_{2} \mathrm{O}$ on the $\mathrm{CO} \mathrm{V}$-T energy transfer have not been investigated by means of the QEPAS so far. Previous studies on CO QEPAS sensors [10] were focused on the improvement of CO detection sensitivities. Therefore, the motivation of this paper is to investigate the V-T relaxation process of $\mathrm{CO}$ and the influence of $\mathrm{H}_{2} \mathrm{O}$ on the $\mathrm{CO}$ V-T relaxation energy transfer in detail using the QEPAS sensor system, which is very important for the QEPAS-based CO sensing. It is well known that CO has a slow $\mathrm{V}-\mathrm{T}$ relaxation. Carbon monoxide is the product of incomplete combustion of organic matter due to insufficient oxygen $\left(\mathrm{O}_{2}\right)$ supply. As a colorless and odorless poisonous gas, $100 \mathrm{ppm}$ level CO can cause danger to the human central nervous system and heart [17]. Therefore, the study of the CO V-T relaxation process is important for the CO QEPAS sensor design and optimization.

Near-IR telecommunication diode lasers have been widely used in spectroscopic technology due to their narrow line-width, fast tuning rate, stable emitting wavelength, and long life [18-20]. Moreover, compared with the mid-infrared quantum cascade lasers (QCLs) or far-infrared Terahertz (THz) lasers [21-23], near-IR telecommunication lasers are more cost-effective. Although the absorption of the overtone band of molecules in the near-IR region is weaker than that of the fundamental band in the mid-IR region, the sensitivity loss can be compensated by boosting the laser power [24-26], due to the fact that PAS sensitivity is proportional to the optical excitation power. In this work, a near-IR QEPAS sensor was developed to investigate the CO V-T relaxation in the dry or wet nitrogen $\left(\mathrm{N}_{2}\right)$ gas mixtures respectively, by use of a $1.57 \mu \mathrm{m}$ near-IR distribute feedback (DFB) laser source. The QEPAS sensor performance under different humidities and pressures were evaluated in detail by experimental investigation and theoretical simulation.

\section{Experimental Setup}

A schematic of the experimental setup is shown in Figure 1. A fiber-coupled distributed feedback (DFB) diode laser with an emitting wavelength centered at $1566.31 \mathrm{~nm}$ (Model PN: DFB-914010C1424-42, Sichuan Tengguang Electronics and Technology Co., Chengdu, China), was used to excite photoacoustic signals.

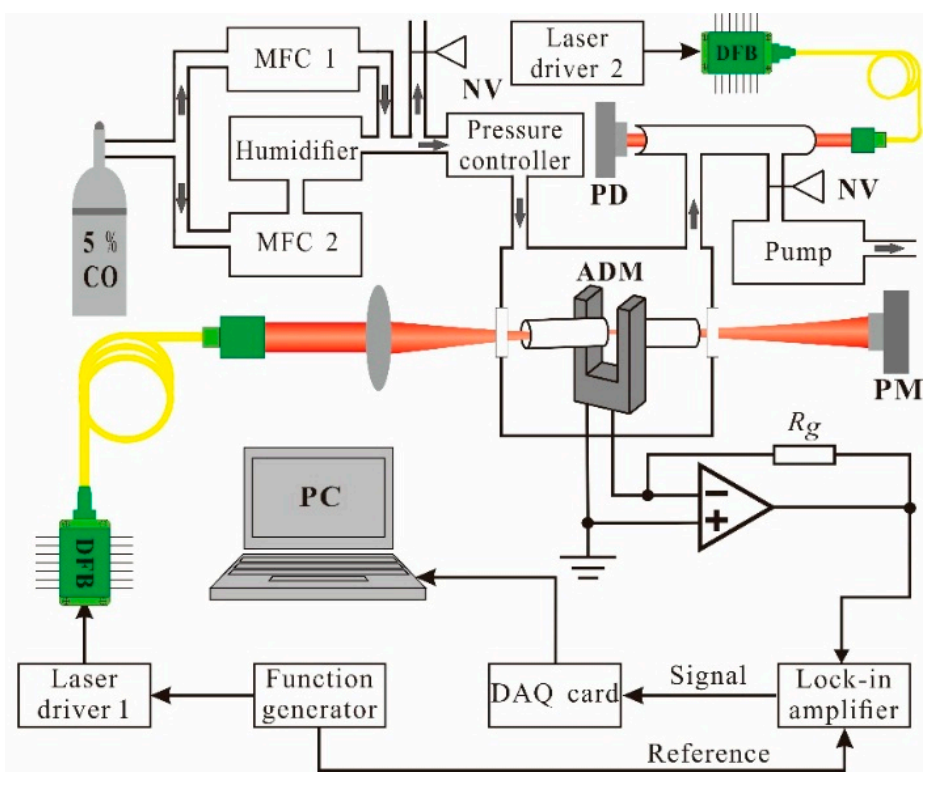

Figure 1. Schematic diagram of the experimental setup. MFC: mass flow controller; PM: power meter; PD: photo detector; NV: needle valve; ADM: acoustic detection module; PC: personal computer. 
The laser current and temperature were controlled by a control electronics unit (CEU) [27]. The current of the DFB laser was modulated at the half resonance frequency of the QTF by the CEU. The laser wavelength can be tuned from $6377.41 \mathrm{~cm}^{-1}$ to $6388.35 \mathrm{~cm}^{-1}$ by changing the laser temperature from $36.45^{\circ} \mathrm{C}$ to $8.45^{\circ} \mathrm{C}$ with a constant current of $100 \mathrm{~mA}$. According to the HITRAN database [28], the molecular absorption lines for $\mathrm{CO}$ and $\mathrm{H}_{2} \mathrm{O}$ within the wavelength range of $6380.5 \mathrm{~cm}^{-1}$ to $6385.5 \mathrm{~cm}^{-1}$ are shown in Figure 2.

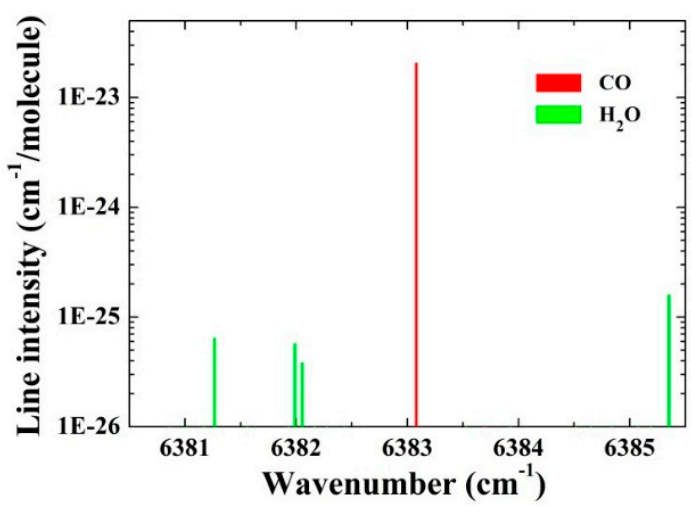

Figure 2. Absorption lines of $\mathrm{CO}$ and $\mathrm{H}_{2} \mathrm{O}$ between $6380.5 \mathrm{~cm}^{-1}$ and $6385.5 \mathrm{~cm}^{-1}$.

The CO absorption line located at $6383.1 \mathrm{~cm}^{-1}$, is two orders of magnitude higher than that of neighbor $\mathrm{H}_{2} \mathrm{O}$ lines, indicating that the target absorption line is interference-free from the $\mathrm{H}_{2} \mathrm{O}$ absorption lines and it can be used to investigate the influence of $\mathrm{H}_{2} \mathrm{O}$ on the $\mathrm{CO} \mathrm{V}-\mathrm{T}$ relaxation using the QEPAS technique. With a constant laser temperature of $21.95^{\circ} \mathrm{C}$, the laser current was scanned from $88 \mathrm{~mA}$ to $108 \mathrm{~mA}$, corresponding to $6383.3 \mathrm{~cm}^{-1}$ to $6382.9 \mathrm{~cm}^{-1}$, in order to cover the selected $\mathrm{CO}$ absorption line. The output laser beam was focused to pass through the QEPAS spectrophone with a beam waist radius of $50 \mu \mathrm{m}$ by a fiber focuser (Model PN: 163426-0, OZ Optics, Ottawa, ON, Canada). A commercially available QTF with a resonance frequency $(f)$ of $32.755 \mathrm{kHz}$ and quality factor ( $Q$-factor) of 13,534 , measured in the atmosphere, was used as the photoacoustic transducer. The QEPAS spectrophone was constructed in an on-beam spectrophone configuration [29], including two identical metallic tubes with the length of $4.0 \mathrm{~mm}$, the inner diameter of $0.8 \mathrm{~mm}$ and the outer diameter of $1.24 \mathrm{~mm}$. The resonance frequency and $Q$-factor of the on-beam QEPAS spectrophone were measured to be $f=32755.4$ and $Q=3432$. The optimum on-beam QEPAS spectrophone can offer a signal-to-noise ratio (SNR) enhancement factor of $\sim 30$. The output signal from the QTF was processed by a low noise trans-impedance amplifier (TPA) with a feedback resistor of $R_{g}=10 \mathrm{M} \Omega$, and then led to a lock-in amplifier (Model: SR830, Stanford Research Systems, Inc., Sunnyvale, CA, USA) to demodulate the signal in $2 f$ mode. The lock-in amplifier was set to a filter slope of $12 \mathrm{~dB} /$ oct and a time constant of $300 \mathrm{~ms}$, which corresponds to a detection bandwidth $\Delta f=0.833 \mathrm{~Hz}$. A computer with a NI DAQ card (Model: NI PCI-6251, National Instrument, Austin, TX, USA) was used to acquire and process all the data of this QEPAS sensor.

The gas sample with $5 \% \mathrm{CO}$ in $\mathrm{N}_{2}$ was divided into two gas lines, which were directed to two mass flow controllers (MFCs) (Model: CS200(A), D07-19B, Beijing Seven Star Electronics Co., Beijing, China), respectively. One gas line controlled by MFC \#2 was humidified by a humidifier (Model: MH-110-24F-4, Perma Pure., Lakewood, NJ, USA). The other gas line controlled by MFC \#1 merged with the humidified gas line to generate gas mixtures with different humidities. The pressure in the system was controlled by a pressure controller (Model: 649B13TS1M22M, MKS Instruments Inc., Andover, MA, USA) and a vacuum pump (Model: D16C, Oerlikon Leybold Vacuum Inc., Cologne, Germany). A 50-cm long gas absorption cell was installed after the acoustic detection module (ADM) to monitor the $\mathrm{H}_{2} \mathrm{O}$ concentration by means of direct absorption spectroscopy implemented by a DFB diode laser (Model: DFB-136813C1424, Chengdu Huawin Laser Co., Sichuan, Chengdu, China,) 
emitting at $7306.75 \mathrm{~cm}^{-1}$ and a photo detector (Model: GT-51084-12N12, GlobTek, Inc., Northvale, NJ, USA). The gas flow rate of the system was set at 70 standard-state cubic centimeters per minute (sccm).

\section{Results and Discussion}

\subsection{Optimization of Modulation Amplitude and Pressure in Dry and Wet Gas Samples}

As the QEPAS is a $2 f$ wavelength modulation based technique, the sensor performance depends on the modulation amplitude of the laser source and the gas pressure. With a low optical excitation power, the QEPAS signal can be expressed as [25]:

$$
S(P)=K \cdot P \cdot C \cdot Q(P) \cdot \alpha_{0}(P) \cdot \varepsilon(P)
$$

where $K, P$ and $C$ are the sensor constant, the incident optical power and the target gas concentration, respectively. These parameters are independent of the pressure $(P)$. The $Q(P), \alpha_{0}(P)$ and $\varepsilon(P)$ are the quality factor ( $Q$-factor) of QTF, the peak of $2 f$ absorption spectrum, and the conversion efficiency $\varepsilon$ of the absorbed optical power into the sound, respectively. $Q, \alpha_{0}$ and $\varepsilon$ are pressure dependent. Moreover, the laser wavelength modulation must match the pressure-dependent absorption linewidth. The highest $\alpha_{0}$ is achieved when the modulation amplitude is close to the absorption linewidth [26]. Therefore, the sensor performance was evaluated by changing the gas pressure of the system and the wavelength modulation amplitude of the laser. Figure $3 a, b$ show that the modulation amplitude of the laser current was optimized at different pressures with dry and humid gas samples, respectively. The $2 f$ QEPAS signal amplitudes for the dry $5 \% \mathrm{CO} / \mathrm{N}_{2}$ mixture are depicted in Figure $3 a$, while the $2 f$ QEPAS signal amplitudes for the $5 \% \mathrm{CO} / \mathrm{N}_{2}$ mixture with $1.6 \%$ water are depicted in Figure $3 \mathrm{~b}$. The water concentration was measured via direct absorption spectroscopy, as shown in Figure 1. The $Q$-factor and the resonance frequency of the QTF were actively measured by the CEU.
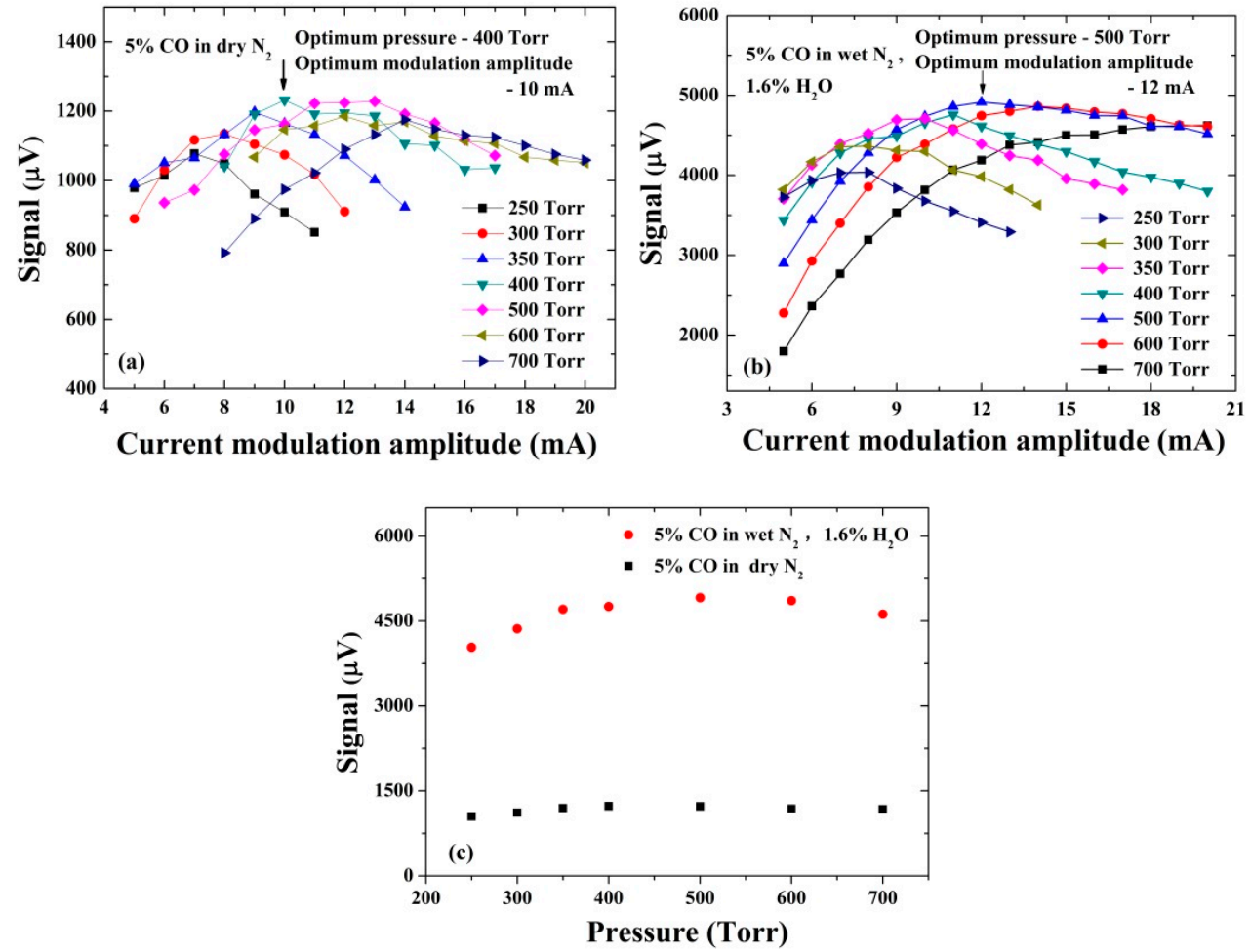

Figure 3. (a,b) $2 f$ QEPAS signal amplitudes obtained at different pressures and laser modulation amplitudes in dry and wet 5\% CO/ $\mathrm{N}_{2}$ mixtures, respectively; (c) $2 f$ QEPAS signal amplitudes for optimum modulation amplitude at different gas pressures. 
Figure 3c shows the dry and wet QEPAS signal amplitudes for the optimized modulation amplitude at different gas pressures. As shown in Figure 3a,c, the maximum QEPAS signal amplitude in the dry $5 \% \mathrm{CO} / \mathrm{N}_{2}$ mixture, is obtained at the pressure of 400 Torr with a modulation amplitude of $10 \mathrm{~mA}$. The variation of QEPAS signals at the pressures range $250-700$ Torr is $<10 \%$. However, a significant increase of the signal amplitude is observed when water is added to the gas mixture. The maximum QEPAS signal amplitude in the wet $5 \% \mathrm{CO} / \mathrm{N}_{2}$ mixture is obtained at 500 Torr with a modulation amplitude of $12 \mathrm{~mA}$, which is $\sim 4$ times higher than that obtained in the dry condition. The significant increase of the $2 f$ QEPAS signal amplitudes indicates that the $\mathrm{H}_{2} \mathrm{O}$ molecules behave as a signal promoter to enhance the QEPAS signal effectively [10,30].

\subsection{Investigation of the $V-T$ Relaxation: $\mathrm{CO}$ in Dry $\mathrm{N}_{2}$}

The $\mathrm{CO}$ has a slow V-T energy relaxation process and as a result, the relaxation rate cannot effectively follow the modulation frequency of $32 \mathrm{kHz}$ in the QEPAS [10,30]. The discrepancy between the V-T relaxation rate and the modulation frequency usually results in the signal amplitude reduction and phase shift. An alternative approach to improve the relaxation rate is to increase the translation motion of the molecules by improving the gas pressure. However, according to Equation (1), the quality factor $Q(P)$, the peak absorption value $\alpha_{0}(P)$, and the conversion efficiency $\varepsilon(P)$ are pressure dependent. In order to further investigate the V-T process of $C O$, it is necessary to study $Q(P), \alpha_{0}(P)$ and $\varepsilon(P)$ first. For simplicity, the sensor constant $K$ was assumed to be 1 in the following sections.

\subsubsection{Q-Factor Measurement}

A QTF oscillator can be equivalent to a RLC series circuit according to the reference [2,31], and the QTF parameters can correspond to equivalent electrical parameters: mass $(m)$ to inductance $(L)$, rigidity $(k)$ to inverse capacity $(1 / C)$ and damping to resistance $(R)$. The $Q(P)$ of the QTF can be described by the expression [2,31]:

$$
Q(p)=\frac{Q_{v a c}}{1+Q_{v a c} a P^{b}}
$$

where $Q_{v a c}$ is the $Q$-factor in vacuum, $P$ is the gas pressure expressed in Torr, $a$ and $b$ are the parameters specific to a particular kind of QTF, respectively. The $Q_{v a c}$ of the used QTF was measured to be 26,029, before removal of the metallic housing of the QTF. The variation of its $Q$-factor as a function of the gas pressures was measured and plotted in Figure 4.

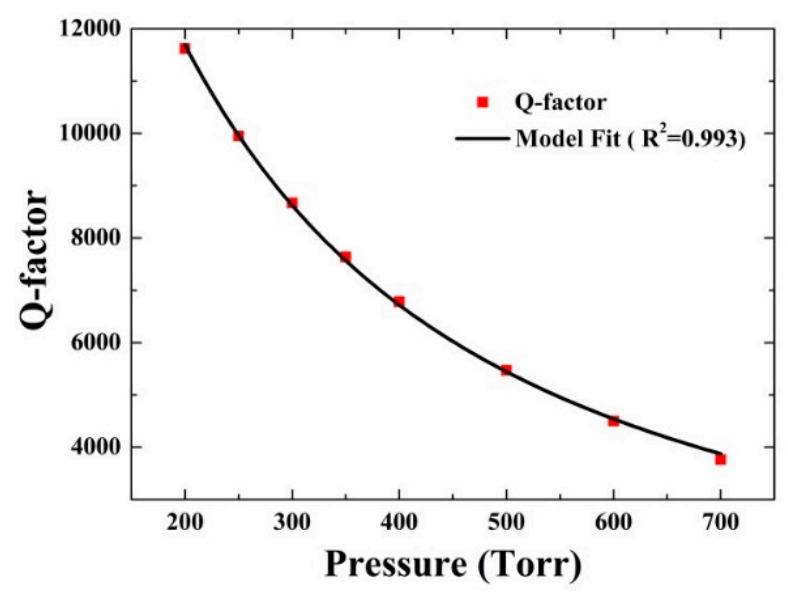

Figure 4. $Q$-factor of the QTF as a function of the gas pressures.

The $Q$-factor decreases monotonously with the increase of the gas pressure. Equation (2) was used to fit the data. The obtained fitting parameters are $a=7.004 \times 10^{-8} \pm 5.749 \times 10^{-9}$ and $b=1.229 \pm 0.014$. The $R$ square of $>0.99$ indicated a good relevancy. 


\subsubsection{Simulation of the Peak $\alpha_{0}(P)$ of $2 f$ Absorption Spectrum}

According to Section 3.1, the pressure $P$ and the laser wavelength modulation amplitude $A$ have a crucial impact on the QEPAS signal amplitude. The simulation of the $\mathrm{CO} 2 f$ wavelength modulation at different pressures can be used to obtain $\alpha_{0}(P)$. The $\mathrm{CO}$ line intensity as well as the broadening coefficients, can be found from the HITRAN database [28]. The self-broadening coefficient of CO was neglected in the simulation, because of its low concentration. The optimum modulation amplitude $A(P)$ and the corresponding $2 f$ absorption peak $\alpha_{0}(P)$ in the gas pressure range $0-1550$ Torr are presented in Figure 5. The numerical calculation indicated that modulation amplitude corresponding to the highest $2 f$ signal is approximately 2.2 times the half width at half maximum (HWHM) of the Lorentzian-shaped absorption line [32,33].

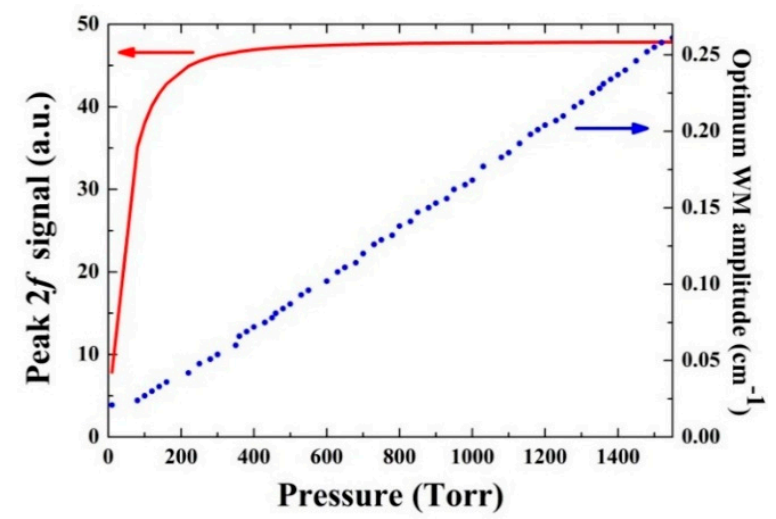

Figure 5. Optimum wavelength modulation amplitude $A(P)$ (circles) and the corresponding $\mathrm{CO} 2 f$ absorption peak $\alpha_{0}(P)$ (line) as a function of the gas pressures.

\subsubsection{Calculation of the Conversion Efficiency $\varepsilon(P)$}

With the obtained $Q(P), \alpha_{0}(P)$ and the experimentally measured signals $S(P)$, the $\varepsilon(P)$ can be calculated according to Equation (1). The measured $S(P)$ with optimum modulation amplitude and calculated $\varepsilon(P)$ at different pressures are plotted in Figure 6. It can be observed that the conversion efficiency increases monotonously with the pressures increasing. This can be attributed to the more drastic collision of molecules at the higher pressure, which means a faster V-T energy transfer. However the maximum signal was obtained at 400 Torr. This is due to the fact that the decrease of the $Q$-factor starts to dominate the $2 f$ signal amplitude beyond 400 Torr although the conversion efficiency between 250 and 700 Torr continues increasing.

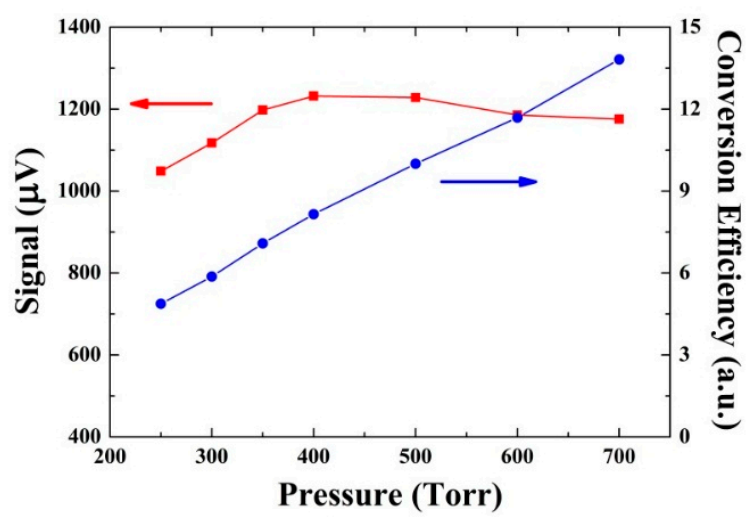

Figure 6. Measured CO QEPAS signals (squares) and corresponding conversion efficiency $\varepsilon(P)$ (circles) as a function of the gas pressure. 
According to reference [32], the conversion efficiency $\varepsilon(P)$ can be expressed as:

$$
\varepsilon(P)=\frac{1}{\sqrt{1+\left[\frac{2 \pi \cdot f \cdot P_{0} \cdot \tau_{0}^{N}{ }^{2}}{P}\right]}}
$$

In order to achieve a linear fit, Equation (3) is rewritten as:

$$
\left[\frac{1}{\varepsilon(P)}\right]^{2}=1+\left(2 \pi \cdot f \cdot P_{0} \cdot \tau_{0}^{N}\right)^{2} \cdot \frac{1}{P^{2}}
$$

where $f$ and $P_{0} \tau_{0}^{N}$ represent the modulation frequency and the V-T relaxation time constant, respectively. In this way, the relaxation time constant can be determined by the slope $\left(2 \pi \cdot f \cdot P_{0} \cdot \tau_{0}^{N}\right)^{2}$. The variation of conversion efficiency $\varepsilon(P)$ as a function of $1 / P^{2}$ is shown in Figure 7. A linear fitting was carried out and an $R$ square value of 0.998 was obtained. The relaxation time constant $P_{0} \tau_{0}^{N}$ of CO calculated from the fitting slope is $9.95 \pm 0.07 \mathrm{~ms}$ Torr in the dry $\mathrm{N}_{2}$, which is $\sim 3$ and $\sim 5$ times slower than the relaxation time constant of $\mathrm{CH}_{4}$ of $2.9 \pm 0.2 \mathrm{~ms}$ Torr and $\mathrm{HCN}$ of $2.2 \pm 0.4 \mathrm{~ms}$ Torr in dry $\mathrm{N}_{2}$, respectively [16,32]. Moreover the V-T relaxation time $\tau_{0}^{N}$ of $C O$ in dry $\mathrm{N}_{2}$ was calculated to be $25 \mu \mathrm{s}$ by dividing the $P_{0} \tau_{0}^{N}$ by the gas pressure $P_{0}$, which is comparable with the QEPAS modulation period of $\sim 30 \mu \mathrm{s}$. This implies that a dry $\mathrm{CO} / \mathrm{N}_{2}$ mixture is not suitable for the QEPAS technique to determine the $\mathrm{CO}$ concentration.

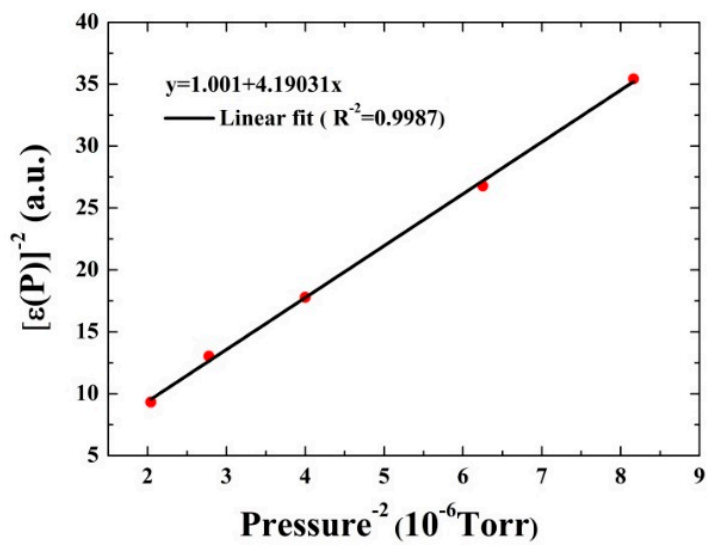

Figure 7. Experimental results (circles) and linear fit for determining the V-T relaxation rate of $\mathrm{CO}$ in dry $\mathrm{N}_{2}$.

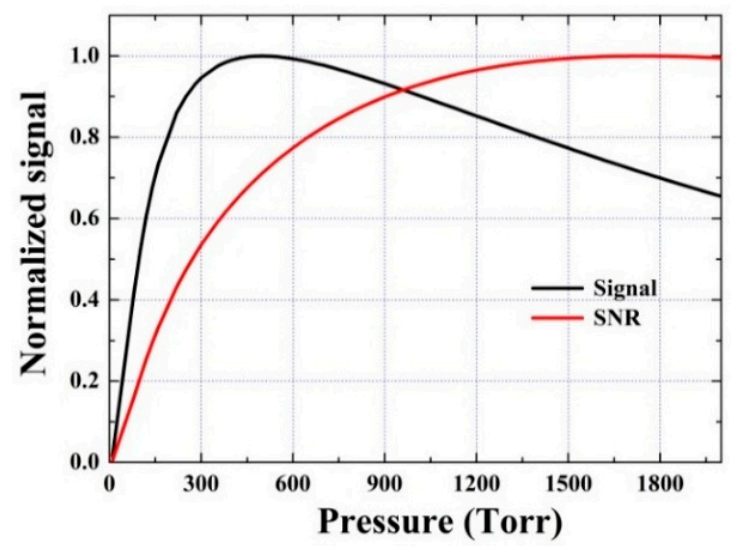

Figure 8. QEPAS sensor performances in terms of signal amplitudes and SNR predicted by the developed relaxation model for detecting $\mathrm{CO}$ in dry $\mathrm{N}_{2}$. 
With the obtained $Q(P), \alpha_{0}(P)$ and $\varepsilon(P)$, Equation (1) can be used to predict the signal $S(P)$ and signal-to-noise ratio $(S N R \sim S(P) / \sqrt{Q(P)})$ at a given concentration of $\mathrm{CO} / \mathrm{N}_{2}$ mixture [29]. The predicted signal amplitudes and the corresponding SNR in the pressure range 0-2000 Torr are presented in Figure 8. The predicted strongest signal is obtained at 500 Torr, which is close to the result of 400 Torr obtained by the developed QEPAS sensor. The highest SNR will be obtained at 1750 Torr according to the developed kinetic model.

\subsection{Investigation of the $V$-T Process: $\mathrm{CO}$ in Wet $\mathrm{N}_{2}$}

In order to investigate the $\mathrm{V}-\mathrm{T}$ relaxation process of $\mathrm{CO}$ in wet $\mathrm{N}_{2}$, a simplified theoretical model of $\mathrm{CO} \mathrm{V}-\mathrm{T}$ relaxation was developed, assuming that only one-stage collisions between molecules occurs [16]. The obtained QEPAS signal in the wet $\mathrm{N}_{2}$ consists of two parts: the collision of $\mathrm{CO}$ and $\mathrm{N}_{2}$ molecules, and $\mathrm{CO}$ and $\mathrm{H}_{2} \mathrm{O}$ molecules, which are described by the signals $S_{1}$ and $S_{2}$, respectively. The signal $S_{2}$ describing the $\mathrm{CO} / \mathrm{H}_{2} \mathrm{O}$ collisions depends on $\mathrm{H}_{2} \mathrm{O}$ partial pressure $P_{H}$. The collisions of two $\mathrm{CO}$ molecules are neglected because of the low concentration of $\mathrm{CO}$. The QEPAS signal with varying $\mathrm{H}_{2} \mathrm{O}$ concentrations, expressed as $S\left(P_{H}\right)=S_{1}+S_{2}$, was measured at 500 Torr. Assuming that the part of the initial vibrational excitation energy released via $\mathrm{CO} / \mathrm{N}_{2}$ collisions $\left(S_{1}\right)$ remains constant, according to Equation (3), the signal $S\left(P_{H}\right)$ can be described as:

$$
S\left(P_{H}\right)=S_{1}+S_{2}=S_{1}\left[1+\frac{\eta-1}{\sqrt{1+\left(\frac{2 \pi \cdot f \cdot P_{0} \cdot \tau_{0}^{H}}{P_{H}}\right)^{2}}}\right]
$$

where $\eta=S(\infty) / S(0), S(\infty)$ and $S(0)$ are equal to the maximum signals obtained with the saturated water and in dry $\mathrm{N}_{2}$ at 500 Torr gas pressure, respectively. Here we assume that the water in the $\mathrm{CO} / \mathrm{N}_{2}$ mixture is saturated, and a $\eta$ value of 4 was obtained. The relaxation time constant $P_{0} \tau_{0}^{H}$ describes the $\mathrm{V}$-T relaxation due to $\mathrm{CO} / \mathrm{H}_{2} \mathrm{O}$ collisions. If $\left(\frac{2 \pi \cdot f \cdot P_{0} \cdot \tau_{0}^{H}}{P_{H}}\right) \gg 1$, Equation (5) can be changed to be a linear equation:

$$
S\left(P_{H}\right) \approx S_{1}\left[1+\frac{\eta-1}{2 \pi \cdot f \cdot P_{0} \cdot \tau_{0}^{H}} P_{H}\right]
$$

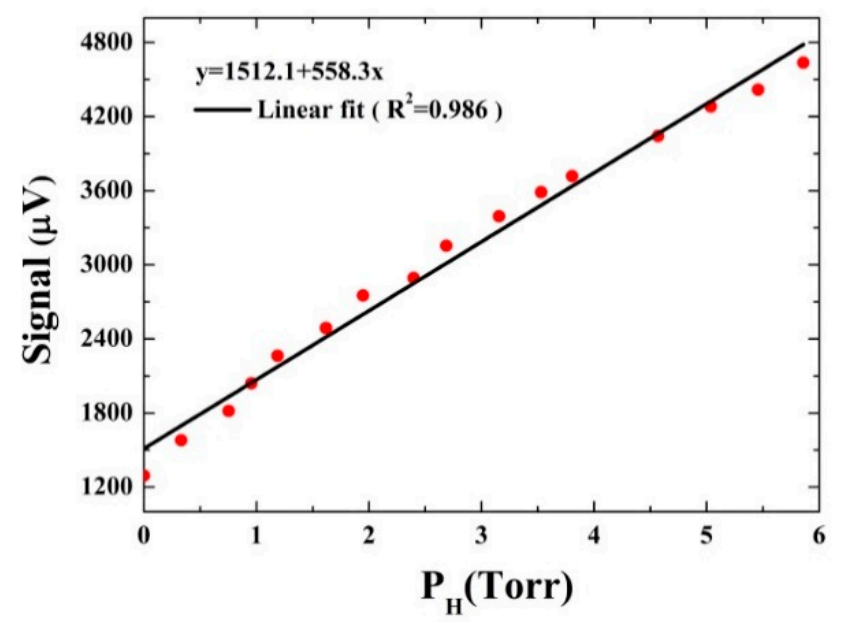

Figure 9. QEPAS signal (circles) as a function of $\mathrm{H}_{2} \mathrm{O}$ concentration for detecting $\mathrm{CO}$ in wet $\mathrm{N}_{2}$. 
The QEPAS signal obtained at different $\mathrm{H}_{2} \mathrm{O}$ partial pressure and the corresponding linear fitting results are shown in Figure 9. A relaxation time constant $P_{0} \tau_{0}^{H}$ of $39.49 \pm 1.95 \mu$ s Torr for CO in wet $\mathrm{N}_{2}$ was calculated from the slope of the fitting curves. And the corresponding V-T relaxation time $\tau_{0}^{H}$ of $0.078 \mu$ s was obtained. Assuming that the influence of water concentrations on $Q(P)$ and $\alpha_{0}(P)$ can be neglected, the conversion efficiency and corresponding QEPAS signals can also be determined at given water concentration and gas pressure, according to Equations (1) and (5).

The profile of QEPAS signals obtained in dry and wet $5 \% \mathrm{CO} / \mathrm{N}_{2}$ mixture are shown in Figure 10. The laser current was scanned from 88 to $108 \mathrm{~mA}$ to cover the $\mathrm{CO}$ absorption line located at $6383.1 \mathrm{~cm}^{-1}$. For the detection of $\mathrm{CO}$ in dry $\mathrm{N}_{2}$, a detection SNR of 22.3 was obtained with 300 ms averaging time at 400 Torr, which corresponds to a normalized noise equivalent absorption coefficient (NNEA) of $5.92 \times 10^{-8} \mathrm{~W} \cdot \mathrm{cm}^{-1} / \mathrm{Hz}^{1 / 2}$. However the detection SNR of 84.2 was obtained in the wet $\mathrm{N}_{2}$ at 500 Torr, which is nearly four times higher than that obtained in the dry $\mathrm{N}_{2}$ and corresponds to a NNEA of $1.556 \times 10^{-8} \mathrm{~W} \cdot \mathrm{cm}^{-1} / \mathrm{Hz}^{1 / 2}$. This is due to the fact that the V-T relaxation time of the CO in wet $\mathrm{N}_{2}$ at 500 Torr was calculated as $0.078 \mu$ s which was shorter than V-T relaxation time of $24.8 \mu$ s obtained in dry $\mathrm{N}_{2}$ at 400 Torr.

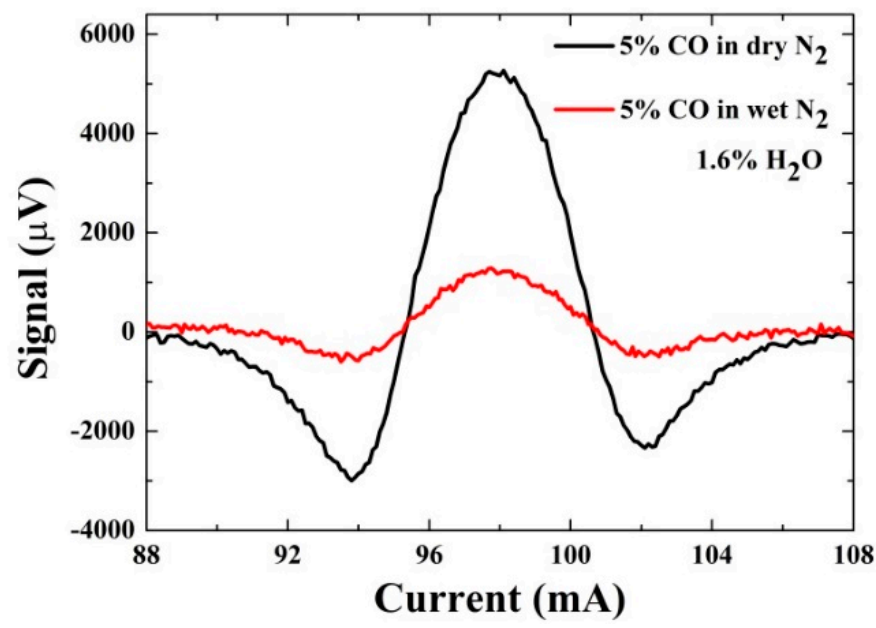

Figure 10. QEPAS signals obtained in the dry $\mathrm{N}_{2}$ and wet $\mathrm{N}_{2}$ as a function a laser current.

\section{Conclusions}

A near-IR QEPAS-based trace gas sensor was developed to investigate the molecular V-T relaxation of $\mathrm{CO}$ by photoacoustic detection. The performance of the QEPAS sensor was evaluated by experimental investigation and theoretical simulation under the different humidity and pressure conditions. A normalized detection sensitivity of $1.556 \times 10^{-8} \mathrm{~W} \cdot \mathrm{cm}^{-1} / \mathrm{Hz}^{1 / 2}$ for $\mathrm{CO}$ in humid gas was achieved when $1.6 \%$ water was added into the gas mixture. The relaxation time constant $P_{0} \tau_{0}^{N}$ in the dry $\mathrm{N}_{2}$ and $P_{0} \tau_{0}^{H}$ in wet $N_{2}$ are $9.95 \pm 0.07 \mathrm{~ms}$ Torr and $39.49 \pm 1.95 \mu \mathrm{s} \mathrm{Torr}$, respectively. The relaxation time constant was improved by as much as a factor of $\sim 230$ with the presence of $1.6 \%$ water. Using Equation (3), the QEPAS signal reached $90 \%$ of its instantaneous-relaxation value when the partial $\mathrm{H}_{2} \mathrm{O}$ pressure was 14 Torr, which corresponds to $60 \%$ relative humidity at $+24{ }^{\circ} \mathrm{C}$. Limited by the performance of our humidifier, our wet gas mixture $(1.6 \%$ water content, $35 \%$ relative humidity at $+24^{\circ} \mathrm{C}$ ) did not reach the $\mathrm{CO}$ instantaneous-relaxation value. The addition of more water can further improve the CO signal amplitude. Due to the crucial role of water in the V-T energy transfer, the water concentration must be controlled or monitored on-line to calibrate the signal amplitude, so that the concentration of the analyzed $\mathrm{CO}$ mixture can be precisely determined.

Acknowledgments: Lei Dong acknowledges support by the 973 program (2012CB921603), the National Natural Science Foundation of China (61575113, 61275213, 61475093, and 61378047), Opened Fund of the State Key Laboratory on Integrated Optoelectronics (IOSKL2015KF25). 
Author Contributions: Xukun Yin, Huadan Zheng and Lei Dong performed the presented measurements, did the data analysis and prepared the manuscript. Xiaoli Liu, Hongpeng Wu and Yanfang Yang did most of the investigations on the topic prior to this work. Weiguang Ma, Lei Zhang, Wangbao Yin, Liantuan Xiao and Suotang Jia revised and improved the manuscript and were involved in the layout of the experiments.

Conflicts of Interest: The authors declare no conflict of interest.

\section{References}

1. Kosterev, A.A.; Bakhirkin, Y.A.; Curl, R.F.; Tittel, F.K. Quartz-enhanced photoacoustic spectroscopy. Opt. Lett. 2002, 27, 1902-1904. [CrossRef] [PubMed]

2. Patimisco, P.; Scamarcio, G.; Tittel, F.K.; Spagnolo, V. Quartz-enhanced photoacoustic spectroscopy: A review. Sensors 2014, 14, 6165-6206. [CrossRef] [PubMed]

3. Dong, L.; Wu, H.P.; Zheng, H.D.; Liu, Y.Y.; Liu, X.L.; Jiang, W.Z.; Zhang, L.; Ma, W.G.; Ren, W.; Yin, W.B.; et al. Double acoustic micro-resonator quartz enhanced photoacoustic spectroscopy. Opt. Lett. 2014, 39, 2479-2482. [CrossRef] [PubMed]

4. Sampaolo, A.; Patimisco, P.; Dong, L.; Geras, A.; Scamarcio, G.; Starecki, T.; Tittel, F.K.; Spagnolo, V. Quartz-enhanced photoacoustic spectroscopy exploiting tuning fork overtone modes. Appl. Phys. Lett. 2015, 107, 231102:1-231102:4. [CrossRef]

5. Zheng, H.D.; Dong, L.; Yin, X.K.; Liu, X.L.; Wu, H.P.; Zhang, L.; Ma, W.G.; Yin, W.B.; Jia, S.T. ppb-level QEPAS $\mathrm{NO}_{2}$ sensor by use of electrical modulation cancellation method with a high power blue LED. Sens. Actuators B Chem. 2015, 208, 173-179. [CrossRef]

6. Wu, H.P.; Dong, L.; Ren, W.; Yin, W.B.; Ma, W.G.; Zhang, L.; Jia, S.T.; Tittel, F.K. Position effects of acoustic micro-resonator in quartz enhanced photoacoustic spectroscopy. Sens. Actuators B Chem. 2015, 206, 364-370. [CrossRef]

7. Jahjah, M.; Jiang, W.; Sanchez, N.P.; Ren, W.; Patimisco, P.; Spagnolo, V.; Herndon, S.C.; Griffin, R.J.; Tittel, F.K. Atmospheric $\mathrm{CH}_{4}$ and $\mathrm{N}_{2} \mathrm{O}$ measurements near Greater Houston area landfills using a QCL-based QEPAS sensor system during DISCOVER-AQ 2013. Opt. Lett. 2014, 39, 957-960. [CrossRef] [PubMed]

8. Liu, K.; Zhao, W.X.; Wang, L.; Tan, T.; Wang, G.S.; Zhang, W.J.; Gao, X.M.; Chen, W.D. Quartz-enhanced photoacoustic spectroscopy of HCN from 6433 to $6613 \mathrm{~cm}^{-1}$. Opt. Commun. 2015, 340, 126-130. [CrossRef]

9. Yi, H.M.; Maamary, R.; Gao, X.M.; Sigrist, M.W.; Fertein, E.; Chen, W.D. Short-lived species detection of nitrous acid by external-cavity quantum cascade laser based quartz-enhanced photoacoustic absorption spectroscopy. Appl. Phys. Lett. 2015, 106, 101109:1-101109:5. [CrossRef]

10. Ma, Y.F.; Lewicki, R.; Razeghi, M.; Tittel, F.K. QEPAS based ppb-level detection of $\mathrm{CO}$ and $\mathrm{N}_{2} \mathrm{O}$ using a high power CW DFB-QCL. Opt. Express 2013, 21, 1008-1019. [CrossRef] [PubMed]

11. Cao, Y.C.; Sanchez, N.P.; Jiang, W.Z.; Griffin, R.J.; Xie, F.; Hughes, L.C.; Zah, C.; Tittel, F.K. Simultaneous atmospheric nitrous oxide, methane and water vapor detection with a single continuous wave quantum cascade laser. Opt. Express 2015, 23, 2121-2132. [CrossRef] [PubMed]

12. Ren, W.; Jiang, W.Z.; Sanchez, N.P.; Patimisco, P.; Spagnolo, V.; Zah, C.-E.; Xie, F.; Hughes, L.C.; Griffin, R.J.; Tittel, F.K. Hydrogen peroxide detection with quartz-enhanced photoacoustic spectroscopy using a distributed-feedback quantum cascade laser. Appl. Phys. Lett. 2014, 104, 041117:1-041117:5. [CrossRef]

13. Pohlkotter, A.; Kohring, M.; Willer, U.; Schade, W. Detection of molecular oxygen at low concentrations using quartz enhanced photoacoustic spectroscopy. Sensors 2010, 10, 8466-8477. [CrossRef] [PubMed]

14. Ba, T.N.; Triki, M.; Desbrosses, G.; Vicet, A. Quartz-enhanced photoacoustic spectroscopy sensor for ethylene detection with a $3.32 \mu \mathrm{m}$ distributed feedback laser diode. Rev. Sci. Instrum. 2015, 86, 02311:1-023111:5.

15. Wysocki, G.; Kosterev, A.A.; Tittel, F.K. Influence of molecular relaxation dynamics on quartz-enhanced photoacoustic detection of $\mathrm{CO}_{2}$ at $\lambda=2 \mu \mathrm{m}$. Appl. Phys. B 2006, 85, 301-306. [CrossRef]

16. Kosterev, A.A.; Mosely, T.S.; Tittel, F.K. Impact of humidity on quartz-enhanced photoacoustic spectroscopy based detection of HCN. Appl. Phys. B 2006, 85, 295-300. [CrossRef]

17. Prockop, L.D.; Chichkova, R.I. Carbon monoxide intoxication: An updated review. J. Neurol Sci. 2007, 262, 122-130. [CrossRef] [PubMed]

18. Ma, Y.F.; Yu, G.; Zhang, J.B.; Yu, X.; Sun, R. Sensitive detection of carbon monoxide based on a QEPAS sensor with a $2.3 \mu \mathrm{m}$ fiber-coupled antimonide diode laser. J. Opt. UK 2015, 17. [CrossRef]

19. Kosterev, A.A.; Tittel, F.K. Ammonia detection using quartz-enhanced photoacoustic spectroscopy with a near-IR telecommunication diode laser. Appl. Opt. 2004, 43, 6213-6217. [CrossRef] [PubMed] 
20. Zheng, H.D.; Dong, L.; Liu, X.L.; Liu, Y.Y.; Wu, H.P.; Ma, W.G.; Zhang, L.; Yin, W.B.; Jia, S.T. Near-IR telecommunication diode laser based double-pass QEPAS sensor for atmospheric $\mathrm{CO}_{2}$ detection. Laser Phys. 2015, 25, 125601:1-125601:5. [CrossRef]

21. Spagnolo, V.; Patimisco, P.; Borri, S.; Scamarcio, G.; Bernacki, B.E.; Kriesel, J. Mid-infrared fiber-coupled QCL-QEPAS sensor. Appl. Phys. B 2013, 112, 25-33. [CrossRef]

22. Spagnolo, V.; Patimisco, P.; Pennetta, R.; Sampaolo, A.; Scamarcio, G.; Vitiello, M.S.; Tittel, F.K. THz Quartz-enhanced photoacoustic sensor for $\mathrm{H}_{2} \mathrm{~S}$ trace gas detection. Opt. Express 2015, 23, 7574-7582. [CrossRef] [PubMed]

23. Borri, S.; Patimisco, P.; Sampaolo, A.; Beere, H.E.; Ritchie, D.A.; Vitiello, M.S.; Scamarcio, G.; Spagnolo, V. Terahertz quartz enhanced photo-acoustic sensor. Appl. Phys. Lett. 2013, 103, 021105:1-021105:4. [CrossRef]

24. Wu, H.P.; Sampaolo, A.; Dong, L.; Patimisco, P.; Liu, X.L.; Zheng, H.D.; Yin, X.K.; Ma, W.G.; Zhang, L.; Yin, W.B.; et al. Quartz enhanced photoacoustic $\mathrm{H}_{2} \mathrm{~S}$ gas sensor based on a fiber-amplifier source and a custom tuning fork with large prong spacing. Appl. Phys. Lett. 2015, 107, 111104:1-111104:2. [CrossRef]

25. Wu, H.P.; Dong, L.; Zheng, H.D.; Liu, X.L.; Ying, X.K.; Ma, W.G.; Zhang, L.; Yin, W.B.; Jia, S.T.; Tittel, F.K. Enhanced near-infrared QEPAS sensor for sub-ppm level $\mathrm{H}_{2} \mathrm{~S}$ detection by means of a fiber amplified 1582 nm DFB laser. Sens. Actuators B Chem. 2015, 221, 666-672. [CrossRef]

26. Wu, H.P.; Dong, L.; Liu, X.L.; Zheng, H.D.; Yin, X.K.; Ma, W.G.; Zhang, L.; Yin, W.B.; Jia, S.T. Fiber-Amplifier-Enhanced QEPAS Sensor for Simultaneous Trace Gas Detection of $\mathrm{NH}_{3}$ and $\mathrm{H}_{2} \mathrm{~S}$. Sensors 2015, 15, 26743-26755. [CrossRef] [PubMed]

27. Spagnolo, V.; Patimisco, P.; Borri, S.; Scamarcio, G.; Bernacki, B.E.; Kriesel, J. Part-per-trillion level SF 6 detection using a quartz enhanced photoacoustic spectroscopy-based sensor with single-mode fiber-coupled quantum cascade laser excitation. Opt. Lett. 2012, 37, 4461-4463. [CrossRef] [PubMed]

28. The HITRAN Database. Available online: http://www.hitran.com (accessed on 6 September 2015).

29. Dong, L.; Kosterev, A.A.; Thomazy, D.; Tittel, F.K. QEPAS spectrophones: Design, optimization, and performance. Appl. Phys. B 2010, 100, 627-635. [CrossRef]

30. Dong, L.; Lewicki, R.; Liu, K.; Buerki, P.R.; Weida, M.J.; Tittel, F.K. Ultra-sensitive carbon monoxide detection by using EC-QCL based quartz-enhanced photoacoustic spectroscopy. Appl. Phys. B 2012, 107, 275-283. [CrossRef]

31. Kosterev, A.A.; Tittel, F.K.; Serebryakov, D.V.; Malinovsky, A.L.; Morozov, I.V. Applications of quartz tuning forks in spectroscopic gas sensing. Rev. Sci. Instrum. 2005, 76. [CrossRef]

32. Kosterev, A.A.; Bakhirkin, Y.A.; Tittel, F.K.; McWhorter, S.; Ashcraft, B. QEPAS methane sensor performance for humidified gases. Appl. Phys. B 2008, 92, 103-109. [CrossRef]

33. Pao, Y.H. Optoacoustic Spectroscopy and Detection, 1st ed.; Academic Press: New York, NY, USA, 1977; pp. 47-76. 